\title{
Theory of the Viscomagnetic Heat Flux
}

\author{
H. Vestner * \\ Huygens Laboratorium der Rijksuniversiteit, Leiden, Nederland \\ (Z. Naturforsch. 31 a, 540-552 [1976] ; received December 22, 1975)
}

\begin{abstract}
The heat transport in the presence of a viscous flow and a magnetic field is studied for a rarefied polyatomic gas enclosed between infinite parallel plates. The total "viscomagnetic heat flux" is the sum of a bulk and a surface heat flux. According to moment equations of the WaldmannSnider kinetic equation, the bulk heat flux is due to gradients of pressure (i. e. second order derivatives of flow velocity) and of tensor polarization. For the tensor polarization a second order differential equation is solved with boundary conditions obtained by Waldmann's thermodynamic method. This method also supplies an ansatz for the surface heat flux. In a thermally isolated box the viscomagnetic heat flux gives rise to a temperature gradient in $y$-direction if the magnetic field is in $z$-direction and the gas is flowing in $x$-direction. The bulk contributions to this transverse temperature gradient have already been given by Levi, McCourt and Beenakker. Now, the surface contributions and the total effect are studied quantitatively for some gases. In particular, it is discussed how a new surface parameter could be determined from future experimental data.
\end{abstract}

\section{Introduction}

Since Maxwell's work ${ }^{1}$ on transport phenomena in rarefied gases it is known that modifications on the heat flux and on the friction pressure tensor occur at low pressures: Second order derivatives of flow velocity contribute to the heat flux ${ }^{2}$, and likewise second gradients of temperature are present in the friction pressure tensor ${ }^{3,4}$. The corresponding expressions for the "viscous heat flux" and the "thermal stresses" for a rarefied polyatomic gas in a magnetic field have been derived by Levi et al. ${ }^{5,6}$. For a polyatomic gas in a magnetic field, the transport processes are no longer isotropic, the second rank thermal conductivity tensor and the fourth rank viscosity tensor replace the scalar transport coefficients: Senftleben-Beenakker effect ${ }^{7}$. Similarly, in rarefied gases the effects due to second order gradients of temperature and velocity are influenced by a magnetic field ${ }^{6}$. In particular, the magnetic field dependent thermal stresses become manifest ${ }^{5,6}$ in the Scott effect ${ }^{8}$ (thermomagnetic torque). As a reciprocal phenomenon, Levi et al. ${ }^{6}$ predicted the existence of a viscomagnetic heat flux: In a rarefied polyatomic gas, flowing in $x$-direction, a heat flux in $y$-direction should occur, if a magnetic field in $z$-direction is present. In a thermally isolated rectangular capillary, a transverse temperature gradient builds up. Recently, the existence of this effect has been demonstrated by Eggermont et al. ${ }^{9}$.

* On leave from Institut für Theoretische Physik der Universität Erlangen-Nürnberg, Erlangen, West Germany. Reprint requests to Dr. H. Vestner, Institut für Theoretische Physik der Universität Erlangen-Nürnberg, Glückstraße 6, D-8520 Erlangen.
Besides the bulk effects, discussed so far, the boundary layer phenomena play an equally important role in a rarefied gas. So, for the thermomagnetic torque, slip effects (thermal ${ }^{5}$ and thermomagnetic ${ }^{10}$ ) have to be taken into account besides the bulk thermal stresses. This is in accordance with the existence of the thermomagnetic pressure difference ${ }^{11,12}$, a phenomenon which is entirely determined by slip effects ${ }^{10,13}$.

The topic of this paper is the calculation of the boundary layer contributions to the viscomagnetic heat flux. Two types of such contributions are discussed. First, it is noted that the total heat transported through the slit formed by two infinite parallel plates consists of the bulk heat flux $\boldsymbol{q}$ and of the "surface heat flux" $Q$. The surface heat flux ${ }^{14-16}$ accounts for the difference between the actual value of the heat flux at the wall and the value of $\boldsymbol{q}$ extrapolated to the wall. Secondly, the bulk heat flux $\boldsymbol{q}$ itself is influenced by boundary effects. In this connection, the behaviour near the wall of the tensor polarization and the Kagan polarization (which are used for the description of the field effects) is of crucial importance. The treatment is based ${ }^{17}$

i) on moment equations derived from the Waldmann-Snider kinetic equation ${ }^{18,19}$, and

ii) on boundary conditions obtained from the interfacial entropy production by Waldmann's thermodynamical method ${ }^{14}$.

In particular, a differential equation and a boundary condition for the tensor polarization, and an ansatz for the surface heat flux are available. 
The transverse temperature gradient calculated in this way consists of the bulk contribution already given by Levi et al. ${ }^{6}$ and of a "surface contribu. tion". For the gas $\mathrm{N}_{2}$ as a representative example, the importance of the surface contribution is studied quantitatively. To this end, for two of the phenomenological surface parameters the values are used which were obtained from the analysis of thermomagnetic pressure difference data ${ }^{12}$. The thermal slip number is taken ${ }^{16}$ from thermal transpiration data. Furthermore, a new surface parameter occurs which characterizes the coupling of the surface heat flux with the boundary value of the Kagan polarization. It is shown how the magnitude of this parameter influences the total effect. Special attention is given to the value and position of the maximum of the transverse temperature gradient as a function of the magnetic field strength for different orientations of the field.

\section{Statement of the Problem}

In a rarefied polyatomic gas which flows through a flat rectangular capillary placed in a magnetic field, a transverse temperature gradient perpendicular to the flow direction occurs. This was predicted by Levi et al. ${ }^{6}$ and recently has experimentally been verified by Eggermont et al. ${ }^{9}$. For simplicity, theoretical calculations will be made for the flow of a gas between infinite parallel plates (of distance $2 d$ ) located at $z= \pm d$, with a pressure gradient

$$
\boldsymbol{\nabla} p^{(0)}=(\partial p / \partial x)_{0} \boldsymbol{e}_{x}
$$

applied (for zero field) in $x$-direction.

The total heat transported parallel to the plates is the sum of a bulk and a surface term,

$$
\boldsymbol{J}_{q}=\int_{-d}^{+d} \mathrm{~d} z \boldsymbol{q}^{\tan }+\boldsymbol{Q}(d)+\boldsymbol{Q}(-d),
$$

with $\boldsymbol{q}^{\text {tan }} \equiv \boldsymbol{q}-\boldsymbol{e}_{z} \boldsymbol{e}_{z} \cdot \boldsymbol{q}$. Here, $\boldsymbol{q}$ is the heat flux in the bulk gas and $\boldsymbol{Q}( \pm d)$ is the surface heat flux at the plates $z= \pm d$. The surface heat flux ${ }^{14}$ makes up for the difference between the true boundary value of the heat flux and the value of $\boldsymbol{q}$ obtained by extrapolation to the wall. From a thermodynamical point of view, the surface heat flux is linked with the thermal slip, i.e. with phenomena like thermal transpiration and thermal force ${ }^{16}$.

The bulk heat flux $\boldsymbol{q}$ is a sum of two terms,

$$
\boldsymbol{q}=-\boldsymbol{\lambda} \cdot \boldsymbol{\nabla} T+\boldsymbol{q}_{\mathrm{VMI}},
$$

the ordinary contribution due to a temperature gradient, and the viscomagnetic bulk heat flux $\boldsymbol{q}_{\mathrm{vM}}$ which arises from a pressure gradient, i. e. from second order derivatives of velocity. The second rank thermal conductivity tensor $\lambda$ depends on the strength and orientation of the magnetic field, with the field influence (Senftleben-Beenakker effect ${ }^{7}$ ) being a small effect of the order of $1 \%$. For zero field, the temperature is constant in plane Poiseuille flow. Hence, due to the smallness of the field dependent temperature gradient, the first term in Eq. (2) can in good approximation be replaced by its isotropic value $-\lambda \nabla T$ :

$$
\boldsymbol{q} \approx-\lambda \nabla T+\boldsymbol{q}_{\mathrm{VII}} .
$$

Under the assumption that $\nabla T$ depends on $z$ only, both $\partial T / \partial x$ and $\partial T / \partial y$ are constant, so Eqs. (1) and (3) give

$$
\boldsymbol{J}_{q} \approx 2 d\left[-\lambda(\boldsymbol{\nabla} T)^{\tan }+\overline{\left.\boldsymbol{q}_{\mathrm{VM}}^{\mathrm{tan}}\right]}+\boldsymbol{Q}(d)+\boldsymbol{Q}(-d),\right.
$$

where

$$
\overline{\boldsymbol{q}_{\mathrm{VM}}^{\mathrm{tan}}}=\frac{1}{2 d} \int_{-d}^{+d} \mathrm{~d} z \boldsymbol{q}_{\mathrm{VM}}^{\mathrm{tan}}
$$

is the value of $\boldsymbol{q}_{\mathrm{VM}}^{\tan }$ averaged over the slit.

If the walls of the channel are adiabatic, the viscomagnetic heat flux gives rise to a transverse temperature gradient. From the adiabatic condition $\boldsymbol{J}_{q}=\mathbf{0}$ and Eq. (4) one finds

$$
(\boldsymbol{\nabla} T)^{\tan }=\frac{1}{\lambda}\left[\overline{\boldsymbol{q}_{\mathrm{VM}}^{\mathrm{tan}}}+\frac{1}{2 d}(\mathbf{Q}(d)+\boldsymbol{Q}(-d))\right] .
$$

In the following sections this temperature gradient shall be calculated in lowest order of the inverse of the average pressure $p_{0}$, viz. in the order $p_{0}{ }^{-1}$. The surface heat flux in this approximation does not contain $(\boldsymbol{\nabla} T)^{\tan }$ itself.

\section{Heat Flux in the Bulk Gas}

In a non-equilibrium situation in a polyatomic gas, the rotational angular momenta $\boldsymbol{J}$ of the molecules, due to the nonspherical interaction, are partially aligned through collisions. For most gases, the (second rank) tensor polarization $\mathbf{a}^{(0200)}$,

$$
\begin{aligned}
a_{\mu \nu}^{(0200)} & =\left\langle\Phi_{\mu \nu}^{(0200)}\right\rangle, \\
\Phi_{\mu \nu}^{(0200)} & =\sqrt{\frac{15}{2}}\left(\left\langle J^{2}\left(J^{2}-\frac{3}{4}\right)\right\rangle_{0}\right)^{-\frac{1}{2}} \overline{J_{\mu} J_{\nu}},
\end{aligned}
$$


and the (third rank) Kagan polarization $\underline{\mathbf{a}}^{(1200)}$,

$$
a_{\lambda \mu \nu}^{(1200)}=\sqrt{\frac{m}{k_{\mathrm{B}} T_{0}}}\left\langle c_{\lambda} \Phi_{\mu \nu}^{(0200)}\right\rangle,
$$

are the dominant types of alignment in streaming and heat conducting gases ${ }^{7}$. The symbol $\langle\ldots\rangle$ denotes a non-equilibrium average, $\langle\ldots\rangle_{0}$ an equilibrium average, respectively; $\rightarrow$ refers to the symmetric traceless part of a tensor; $m, \mathbf{c}$ are the molecular mass and velocity, $k_{\mathrm{B}}$ is Boltzmann's constant and $T_{0}$ the equilibrium temperature. Application of the moment method ${ }^{20}$ to the relevant kinetic equation due to Waldmann ${ }^{18}$ and Snider ${ }^{19}$ leads to a set of coupled transport relaxation equations for these polarizations and other physical quantities of interest, like the heat flux $\boldsymbol{q}$ and the friction pressure tensor $\overline{\mathbf{p}}$. So, the following ansatz for the bulk viscomagnetic heat flux is obtained:

$$
\begin{aligned}
\boldsymbol{q}_{\mathrm{VMI}}= & \lambda \alpha T_{0} p_{0}{ }^{-1}\left(\boldsymbol{\delta}-\Psi_{1} \boldsymbol{\Lambda}\left(\varphi_{12}\right)\right) \cdot \boldsymbol{\nabla} p \\
& +\lambda \Psi_{2} T_{0} p_{0}{ }^{-1} \boldsymbol{\nabla} \cdot \sum_{n=-2}^{+2}\left(1+i n \varphi_{12}\right)^{-1} \mathbf{A}_{n}{ }^{(02)} .
\end{aligned}
$$

The first term in Eq. (8) is easily recognized as the generalization of Maxwell's viscous heat flux. The factor $\frac{2}{5}$ in Maxwell's work ${ }^{1}$ is replaced by

$$
\alpha=\frac{2}{5} \frac{\lambda_{\mathrm{tr}}}{\lambda}(1+\varepsilon),
$$

i. e. in a polyatomic gas the translational part $\lambda_{\text {tr }}$ of the total heat conductivity $\lambda$ occurs. If values for the viscosity $\eta$ and the bulk viscosity $\eta_{\mathrm{V}}$ are known, the ratio $\lambda_{\text {tr }} / \lambda$ can be calculated from

$$
\begin{aligned}
\frac{\lambda_{\mathrm{tr}}}{\lambda}=\left(\frac{15}{4} \frac{k_{\mathrm{B}}}{m} \frac{\eta}{\lambda}+\frac{5}{6} c_{\mathrm{r}}\left(1-c_{\mathrm{r}}\right) \frac{\eta}{\eta_{\mathrm{V}}}\right) \\
/\left(1+\frac{5}{6} c_{\mathrm{r}} \frac{\eta}{\eta_{\mathrm{V}}}\right),
\end{aligned}
$$

where $c_{\mathrm{r}}=c_{\text {rot }} /\left(c_{\text {rot }}+\frac{3}{2} k_{\mathrm{B}}\right)$ is the relative rotational specific heat. The correction term $\varepsilon$ arises from the coupling of (eliminated) higher moments, e.g. $\mathbf{a}^{(2010)}$ and $\mathbf{a}^{(2001)}$. If the contributions due to inelastic collisions (i. e. change of rotational energy) are neglected, $\varepsilon$ can be expressed ${ }^{21}$ in terms of Chapman-Cowling reduced $\Omega$-integrals and is approximately given by

$$
\varepsilon \approx \frac{\frac{7}{2} \Omega^{(2,2) *}-4 \Omega^{(2,3) *}}{\frac{43}{12} \Omega^{(2,2) *}-4 \Omega^{(2,3) *}+\frac{20}{7} \Omega^{(2,4) *}} .
$$

Special values are $\varepsilon=0$ for Maxwell molecules and $\varepsilon=-0.2049$ for hard spheres. The hard sphere value is close to the numbers obtained (with different types of approximation) by Jones ${ }^{3}$ and by Burnett ${ }^{4}$. For a Lennard-Jones potential, $\varepsilon$ is a function of the reduced temperature ${ }^{22}$; typical values for gases like $N_{2}$ at room temperature are $\varepsilon \approx-0.1$.

A magnetic field $\boldsymbol{H}=H \boldsymbol{h}(\boldsymbol{h} \cdot \boldsymbol{h}=1)$ influences the transport phenomena through the molecular magnetic moment $g_{\mathrm{r}} \mu_{\mathrm{N}} \boldsymbol{J}$ connected with the rotation, $g_{\mathrm{r}}$ is the rotational $g$-factor, $\mu_{\mathrm{N}}$ the nuclear magneton. The second rank tensor $\boldsymbol{\Lambda}$ in Eq. (8),

$$
\begin{aligned}
\Lambda_{\mu \nu}\left(\varphi_{12}\right)= & h_{\mu} h_{\nu} f\left(\varphi_{12}\right) \\
& +\left(\delta_{\mu \nu}-h_{\mu} h_{v}\right) \frac{1}{2}\left(f\left(\varphi_{12}\right)+2 f\left(2 \varphi_{12}\right)\right) \\
& +\varepsilon_{\mu \lambda \nu} h_{\lambda} \frac{1}{2}\left(g\left(\varphi_{12}\right)+2 g\left(2 \varphi_{12}\right)\right),
\end{aligned}
$$

with

$$
f\left(\varphi_{12}\right)=\varphi_{12}^{2} /\left(1+\varphi_{12}^{2}\right), g\left(\varphi_{12}\right)=\varphi_{12} /\left(1+\varphi_{12}^{2}\right),
$$

contains the field direction $\boldsymbol{h}$ and depends on the field strength $H$ via the precession angle $\varphi_{12} \propto H / p_{0}$. The precession angle

$$
\varphi_{p q} \equiv \frac{\omega_{\mathrm{H}}}{n_{0} v_{\mathrm{rel}} \Xi\left(\begin{array}{c}
p q 00 \\
p q 00
\end{array}\right)}, \quad v_{\mathrm{rel}}=\left(16 k_{\mathrm{B}} T_{0} / m\right)^{\frac{1}{2}},
$$

is the ratio of the precession frequency $\omega_{\mathrm{H}}=$ $(1 / \hbar) g_{\mathrm{r}} \mu_{\mathrm{N}} H$ and a gas collision frequency; $n_{0}$ is the equilibrium density, $p_{0}=n_{0} k_{\mathrm{B}} T_{0}$. Here, $\varphi_{12}$ and $\varphi_{02}$ will occur. With the help of $\Lambda$, the heat conductivity tensor $\lambda$ can be written as ${ }^{23}$

$$
\boldsymbol{\lambda}=\lambda\left(\boldsymbol{\delta}-\Psi^{(\lambda)} \boldsymbol{\Lambda}\left(\varphi_{12}\right)\right),
$$

where $\Psi^{(\lambda)}$ characterizes the relative size of the field effect *.

The second term in Eq. (8) is due to gradients of the tensor polarization. In the following, for convenience, the variables $\mathbf{A}^{(02)}$ and $\underline{\mathbf{A}}^{(12)}$ will be used instead of $\mathbf{a}^{(0200)}$ and $\underline{\mathbf{a}}^{(1200)}$ :

$\mathbf{A}^{(02)}=\sqrt{2} \Im\left(\begin{array}{l}0200 \\ 0200\end{array}\right) \Im^{-1}\left(\begin{array}{c}2000 \\ 0200\end{array}\right) p_{0} \mathbf{a}^{(0200)}$,

$\underline{\mathbf{A}}^{(12)}=\left[2 \Subset\left(\begin{array}{c}0200 \\ 0200\end{array}\right) \Im\left(\begin{array}{c}1200 \\ 1200\end{array}\right)\right]^{\frac{1}{2}} \Im^{-1}\left(\begin{array}{l}2000 \\ 0200\end{array}\right) p_{0} \underline{\mathbf{a}}^{(1200)}$.

The spherical components $\mathbf{A}_{n}{ }^{(02)} \equiv \mathscr{P}(n): \mathbf{A}^{(02)}$ of the "tensor polarization" $\mathbf{A}^{(02)}=\sum_{n=-2}^{+2} \mathbf{A}_{n}(02)$ itself are determined by velocity gradients (see Section 4). Notice, that the fourth rank projection tensors ${ }^{25}$

* For $\Psi(\lambda)$ see e. g. Refs. ${ }^{23,}{ }^{24}$; with the constants used here, one has

$\Psi(\lambda)=2 \lambda\left(\frac{k \mathrm{~B}}{m} \eta\right)^{-1} \Xi_{\left(\begin{array}{l}1200 \\ 1200\end{array}\right)} \Xi_{\left(\begin{array}{c}0200 \\ 0200\end{array}\right)} \Im^{-2}\left(\begin{array}{c}2000 \\ 2000\end{array}\right) \Psi_{2}^{2} / \Psi(\eta)$,

see Eqs. (16), (18) and (4.1) for $\Psi_{2}$ and $\Psi(\eta)$. The notation for the effective cross sections $\widetilde{S}\left(\begin{array}{c}p \\ p(q) q^{\prime} r^{\prime} s_{1}\end{array}\right)$ is the same as in Reference ${ }^{24}$. 
$\mathscr{P}{ }^{(n)}$ depend on the field direction $\boldsymbol{h}$. The relative size of the two field dependent terms in the bulk viscomagnetic heat flux is determined by the values of the two constants $\Psi_{1}$ and $\Psi_{2}$ which can be expressed in terms of effective cross sections in the following way:

with

$$
\Psi_{1}=f_{1} \Psi_{0}, \Psi_{2}=\frac{2}{5}\left(\lambda_{\mathrm{tr}} / \lambda\right) \Psi_{0},
$$

$$
\begin{aligned}
& f_{1}=\sqrt{\frac{4}{5} \frac{\Im}{\mathfrak{S}\left(\begin{array}{c}
0200 \\
02000
\end{array}\right)}\left(\begin{array}{l}
2000 \\
0200
\end{array}\right)} \\
& \cdot\left[-\Im\left(\begin{array}{l}
1010 \\
1200
\end{array}\right) \Im\left(\begin{array}{l}
1001 \\
1001
\end{array}\right)+\Im\left(\begin{array}{l}
1001 \\
1200
\end{array}\right) \Im\left(\begin{array}{l}
1010 \\
1001
\end{array}\right)\right] \\
& \cdot\left[\Im\left(\begin{array}{l}
1010 \\
1010
\end{array}\right) \Im\left(\begin{array}{l}
1001 \\
1001
\end{array}\right)-\Im^{2}\left(\begin{array}{l}
1010 \\
1001
\end{array}\right)\right]^{-1} \text {, }
\end{aligned}
$$

and

$$
\begin{aligned}
& \Psi_{0}=\sqrt{\frac{5}{4}} \frac{\Im\left(\begin{array}{l}
2000 \\
0200
\end{array}\right)}{\Im\left(\begin{array}{l}
0200 \\
0200
\end{array}\right) \subseteq\left(\begin{array}{l}
1200 \\
1200
\end{array}\right)} \\
& {\left[-\Im\left(\begin{array}{l}
1010 \\
1200
\end{array}\right)+\Im\left(\begin{array}{l}
1001 \\
1200
\end{array}\right) \frac{\Im\left(\begin{array}{l}
1010 \\
1001
\end{array}\right)+\sqrt{\frac{2}{5} \frac{c_{\mathrm{rot}}}{k_{\mathrm{B}}}} \Im\left(\begin{array}{l}
1010 \\
1010
\end{array}\right)}{\Im\left(\begin{array}{l}
1001 \\
1001
\end{array}\right)+\sqrt{\frac{2}{5} \frac{c_{\mathrm{rot}}}{k_{\mathrm{B}}}} \Im\left(\begin{array}{l}
1010 \\
1001
\end{array}\right)}\right] .}
\end{aligned}
$$

According to Eq. (5), the transverse temperature gradient is determined by the averaged viscomagnetic heat flux. With the assumption that the tensor polarization depends on $z$ only, the integration of Eq. (8) gives

$$
\begin{aligned}
\overline{\boldsymbol{q}}_{\mathrm{VM}}= & \lambda \alpha T_{0} p_{0}{ }^{-1}\left[\overline{\boldsymbol{\nabla} p}-\Psi_{1} \boldsymbol{\Lambda}\left(\varphi_{12}\right) \cdot \boldsymbol{\nabla} p^{(0)}\right] \\
& +\lambda \Psi_{2} T_{0} p_{0}{ }^{-1} \frac{1}{2 d} \boldsymbol{e}_{z} \cdot \sum_{n=-2}^{+2}\left(1+i n \varphi_{12}\right)^{-1} \\
& \cdot\left[\mathbf{A}_{n}{ }^{(02)}(d)-\mathbf{A}_{n}{ }^{(02)}(-d)\right] .
\end{aligned}
$$

For the evaluation of the first term in Eq. (19), the magnetic field depend pressure gradient in the streaming gas has to be known. In the second term however, the field free value $\boldsymbol{\nabla} p^{(0)}$ can be inserted, if contributions of the order $\Psi_{1} \Psi^{(\eta)}$ are neglected. The third term in Eq. (19) is given by the boundary values of the tensor polarization, hence boundary layer phenomena will be of importance for the average viscomagnetic heat flux in the bulk.

\section{Boundary Conditions, Surface Heat Flux}

For the calculation of the transverse temperature gradient, boundary conditions for the tensor polarization and an ansatz for the surface heat flux are needed. Both are derived by the thermodynamical method introduced by Waldmann ${ }^{14}$. Starting point is the entropy production at the interface between two media, i. e. the gas and a solid. According to the principles of non-equilibrium thermodynamics, the surface entropy production is transformed into a sum of products of independent "fluxes" and "forces", using the local conservation laws at the interface. In particular, the energy law,

$$
\left(\boldsymbol{q}-\boldsymbol{q}_{\mathrm{s}}\right) \cdot \boldsymbol{n}+\boldsymbol{k} \cdot \boldsymbol{v}=\boldsymbol{\nabla}^{\sigma} \cdot \boldsymbol{Q},
$$

connects the discontinuity of the normal heat fluxes in the gas $(\boldsymbol{q})$ and in the solid $\left(\boldsymbol{q}_{\mathrm{s}}\right)$, and the work term $(\boldsymbol{k} \cdot \boldsymbol{v})$ with the divergence of the surface heat flux $\boldsymbol{Q}$. Here, $\boldsymbol{n}$ is the outward unit normal of the gas at the interface, $\boldsymbol{k}=\mathbf{p} \cdot \boldsymbol{n}$ is the force the gas exerts per unit area of the solid, and $\boldsymbol{\nabla}^{\sigma}$ denotes a two dimensional surface derivative. Linear relations set up between "fluxes" and "forces" supply the boundary conditions ${ }^{14,15}$. So, for the surface heat flux an ansatz is obtained which is coupled with two other vectorial conditions ${ }^{17}$, viz. the velocity slip condition and the boundary condition for the tensor polarization components $\boldsymbol{a}^{(0200)} \equiv\left(\mathbf{a}^{(0200)} \cdot \boldsymbol{n}\right)^{\tan }$ :

$$
\begin{aligned}
& -T_{0}{ }^{-1} \boldsymbol{Q}=C_{\mathrm{h}} l \lambda T_{0}{ }^{-1} \nabla^{\tan \frac{1}{2}}\left(\tilde{T}+T_{\mathrm{s}}\right) \\
& +C_{\mathrm{hm}} \lambda p_{0}{ }^{-1} \boldsymbol{k}^{\mathrm{tan}}+C_{\mathrm{ha}} \lambda \boldsymbol{a}^{(1200)}, \\
& \tilde{\boldsymbol{v}}^{\tan }=C_{\mathrm{mh}} \lambda p_{0}{ }^{-1} \nabla^{\tan } \frac{1}{2}\left(\tilde{T}+T_{\mathrm{s}}\right) \\
& +C_{\mathrm{m}} l \eta^{-1} \boldsymbol{k}^{\mathrm{tan}}+C_{\mathrm{ma}} c_{0} \boldsymbol{a}^{(1200)}, \\
& \boldsymbol{a}^{(0200)}=C_{\mathrm{ah}} \lambda\left(2 p_{0} c_{0}\right)^{-1} \nabla^{\tan } \frac{1}{2}\left(\tilde{T}+T_{\mathrm{s}}\right) \\
& +C_{\mathrm{am}}\left(2 p_{0}\right)^{-1}+C_{\mathrm{a}} \boldsymbol{a}^{(1200)} \text {. }
\end{aligned}
$$

Here, $\boldsymbol{a}^{(1200)}$ stands for $a_{v}^{(1200)}=\left(a_{\lambda \mu \nu}^{(1200)} n_{\lambda} n_{\mu}\right)^{\tan }$, and $l$ is a viscosity characteristic free path,

$$
l=\eta c_{0} p_{0}^{-1}, \quad c_{0}=\left(k_{\mathrm{B}} T_{0} / m\right)^{\frac{1}{2}} .
$$

Typically for a consistent treatment of the rarefied gas, the tilded velocity $\tilde{\boldsymbol{v}}$ and tilded temperature $\tilde{T}$ occur in the boundary conditions instead of the actual velocity and temperature ${ }^{15,16}$,

$$
\begin{aligned}
& \tilde{\boldsymbol{v}}=\boldsymbol{v}+\frac{2}{5}(1+\varepsilon) p_{0}{ }^{-1} \boldsymbol{q}_{\mathrm{tr}}, \\
& \tilde{T}=T+\frac{2}{5}(1+\varepsilon) c_{\mathrm{t}} \boldsymbol{T}_{\mathbf{0}}{p_{\mathbf{0}}}^{-1} \boldsymbol{n} \cdot \overline{\mathbf{p}} \cdot \boldsymbol{n},
\end{aligned}
$$

where $\boldsymbol{q}_{\mathrm{tr}}$ is the translational heat flux, and $c_{\mathrm{t}}=$ $\frac{3}{2} k_{\mathrm{B}} /\left(c_{\mathrm{rot}}+\frac{3}{2} k_{\mathrm{B}}\right)$ is the relative translational specific heat. The phenomenological coefficients $C_{\text {.. }}$ are subject to two restrictive conditions. One is a consequence of the second law of thermodynamics, requiring a positive surface entropy production, i.e. a positive definite $C_{\text {.. }}$-matrix :

$$
\begin{aligned}
& C_{k}>0, \\
& C_{k} C_{l}-\xi^{(k l)}\left(\frac{C_{k l}+C_{l k}}{2}\right)^{2}>0, \quad l, k=\mathrm{a}, \mathrm{h}, \mathrm{m} .
\end{aligned}
$$


The factors $\xi^{(k l)}=\xi^{(l k)}>0$,

$$
\xi^{(\mathrm{mh})}=\lambda\left(\frac{k_{\mathrm{B}}}{m} \eta\right)^{-1}, \quad \xi^{(\mathrm{ma})}=\frac{1}{2}, \quad \xi^{(\mathrm{ha})}=\frac{1}{2} \xi^{(\mathrm{mh})},
$$

enter automatically through the use of the dimensionless slip numbers $C_{\text {.. }}$ instead of the coefficients $\mathrm{L}_{\text {.. }}^{(\mathrm{V})}$ of Ref. ${ }^{17}$. A second requirement stems from Onsager relations:

$$
\begin{aligned}
& C_{\mathrm{ma}}=-C_{\mathrm{am}}, \quad C_{\mathrm{ha}}=-C_{\mathrm{ah}}, \\
& C_{\mathrm{mh}}=C_{\mathrm{hm}} \equiv \sigma .
\end{aligned}
$$

With the anti-symmetry (27), Eq. (25) yields nothing new beyond $\mathrm{Eq}$. (24), whereas the combination of the symmetry (28) with (25) gives a restriction ${ }^{16}$ for the absolute value of the thermal slip number $\sigma$,

$$
C_{\mathrm{m}} C_{\mathrm{h}}-\xi^{(\mathrm{mh})} \sigma^{2}>0 \text {. }
$$

Next, the general boundary conditions $(21)-(23)$ are discussed briefly. According to Eq. (21), the surface heat flux $\boldsymbol{Q}$ is determined by temperature gradients along the wall, by tangential stresses and by the Kagan polarization component $\boldsymbol{a}^{(\mathbf{1 2 0 0 )}}$. For the tangential velocity in Eq. (22), there are several types of slip: two sources of thermal slip, due to a tangential heat flux (hidden in $\tilde{\boldsymbol{v}}$ ) and to tangential temperature gradients, the mechanical slip $\left(\propto C_{\mathrm{m}} \boldsymbol{k}^{\mathrm{tan}}\right)$ and the thermomagnetic slip ${ }^{10,17}$ $\left(\propto C_{\mathrm{ma}} \boldsymbol{a}^{(1200)}\right)$. The boundary value of the tensor polarization component $\boldsymbol{a}^{(\mathbf{0 2 0 0})}$ results from two production mechanisms, characterized by $C_{\text {ah }}$ and $C_{\text {am }}$, and an accommodation process $\left(\propto C_{\mathrm{a}} \boldsymbol{a}^{(1200)}\right)$. Values for the six independent slip numbers can be determined in the following way: $C_{\mathrm{m}}$ is found from deviations of the frictional force from Stokes' formula, $\sigma$ from thermal transpiration in the high pressure limit, and then $C_{\mathrm{h}}$ is obtained from the analysis of thermal force data ${ }^{16}$. Numerical values for $C_{\mathrm{a}}$ and $C_{\text {am }}$ are derived from Knudsen corrections for the field effect of viscosity ${ }^{26}$ and from thermomagnetic pressure difference data ${ }^{12}$. The only coefficient not yet known, $C_{\mathrm{ha}}=-C_{\mathrm{ah}}$, can hopefully be inferred from viscomagnetic heat flux experiments.

Now, the general boundary conditions (21) and (23) shall be specialized to the case of a flowing gas. For the approximate calculation of the polarizations, the first term in Eq. (23) can be neglected since it is nonzero only in the presence of a field, thus it is an order of magnitude smaller than the two remaining terms. Similarly, the tangential force $\boldsymbol{k}^{\tan }$ can be replaced by its "isotropic" (field free) value $\boldsymbol{k}_{0}{ }^{\text {tan }}$. In addition to Eq. (23), there are also boundary conditions for the surface scalar part $\boldsymbol{n n}: \mathbf{a}^{(0200)}$ and the surface tensor part $\mathfrak{a}^{(0200)}$ of the tensor polarization ${ }^{17}$. However it is assumed that they are related by the same $C_{\mathrm{a}}$ to the corresponding components of the normal Kagan polarization $n_{\lambda} a_{\lambda \mu \nu}^{(1200)}$. So, for the present situation one has

$$
\begin{aligned}
\boldsymbol{n} \boldsymbol{n}: \mathbf{a}^{(0200)} & =C_{\mathrm{a}} \boldsymbol{n} \boldsymbol{n}:\left(\boldsymbol{n} \cdot \underline{\mathbf{a}}^{(1200)}\right), \\
\mathfrak{a}^{(0200)} & =C_{\mathrm{a}} \mathfrak{a}^{(1200)} .
\end{aligned}
$$

Thus, a boundary condition for the total tensor $\mathbf{a}^{(0200)}$ can be stated; for the variables $\mathbf{A}^{(02)}$ and $\underline{\mathbf{A}}^{(12)}$ introduced in Eq. (15), it reads

$$
\mathbf { A } ^ { ( 0 2 ) } = \tilde { C } _ { \mathrm { am } } 2 \longdiv { \boldsymbol { k } _ { 0 } { } ^ { \operatorname { t a n } } \boldsymbol { n } } + \tilde { C } _ { \mathrm { a } } \boldsymbol { n } \cdot \underline { \mathbf { A } } ^ { ( 1 2 ) },
$$

where $\tilde{C}_{\mathrm{am}}, \tilde{C}_{\mathrm{a}}$ are related to $C_{\mathrm{am}}, C_{a}$ by

$$
\begin{aligned}
& C_{\mathrm{am}}\left.=\sqrt{2} \underset{\Im}{\Im\left(\begin{array}{c}
2000 \\
0200
\end{array}\right)} \tilde{C}_{0200}\right) \\
& C_{\mathrm{am}}, \\
& C_{\mathrm{a}}=\left[\Im\left(\begin{array}{c}
1200 \\
1200
\end{array}\right) / \widetilde{S}\left(\begin{array}{c}
0200 \\
0200
\end{array}\right)\right]^{\frac{1}{2}} \tilde{C}_{\mathrm{a}} .
\end{aligned}
$$

In the ansatz (21) for the surface flux, the first term can be neglected since it is of order $p_{0}{ }^{-2}$ and here only terms of the order $p_{0}{ }^{-1}$ shall be retained. Using $\tilde{C}_{\mathrm{ha}}$ instead of $C_{\mathrm{ha}}$,

$$
C_{\mathrm{ha}}=\sqrt{2}\left[\Im\left(\begin{array}{l}
0200 \\
0200
\end{array}\right) \Im\left(\begin{array}{l}
1200 \\
1200
\end{array}\right)\right]^{\frac{1}{2}} \Im^{-1}\left(\begin{array}{l}
2000 \\
0200
\end{array}\right) \Psi_{2} \tilde{C}_{\mathrm{ha}},
$$

one finds

$$
\boldsymbol{Q}=-\lambda T_{0} p_{0}{ }^{-1}\left(\sigma \boldsymbol{k}+\tilde{C}_{\mathrm{ha}} \Psi_{2} \boldsymbol{n} \boldsymbol{n}: \underline{\mathbf{A}}^{(12)}\right)^{\mathrm{tan}} .
$$

For the explicit calculation of $\boldsymbol{Q}$, the magnetic field dependent force $\boldsymbol{k}^{\text {tan }}$ (i. e. the pressure gradient) and the Kagan polarization have to be determined.

\section{Tensor Polarization in a Streaming Gas}

By insertion of the ansatz for the Kagan polarization into the moment equation for the tensor polarization, second order differential equations for the spherical components $\mathbf{A}_{n}{ }^{(02)}$ are obtained ${ }^{17}$. For a streaming gas with $\mathbf{A}_{n}{ }^{(02)}=\mathbf{A}_{n}{ }^{(02)}(z)$ they are

$$
\begin{aligned}
\left(1-L_{n}{ }^{2} \frac{\mathrm{d}^{2}}{\mathrm{~d} z^{2}}\right) \mathbf{A}_{n}{ }^{(02)}= & \frac{2 \eta}{1+i n \varphi_{02}}(\overline{\boldsymbol{\nabla} \boldsymbol{v}})_{n} \\
= & \frac{2 z}{1+i n \varphi_{02}}\left(\widehat{\boldsymbol{e}_{z} \boldsymbol{\nabla} p^{(0)}}\right)_{n}, \\
& n=0, \pm 1, \pm 2 .
\end{aligned}
$$

Here, the velocity gradient has been replaced by its field-free value for plane Poiseuille flow. The mag- 
netic field dependent quantity $L_{n}$ has the dimension of a length

$$
L_{n}=L_{0}\left[\left(1+i n \varphi_{02}\right)\left(1+i n \varphi_{12}\right)\right]^{-\frac{1}{2}},
$$

where the free path $L_{0}$,

$$
\begin{aligned}
L_{0} & =n_{0}{ }^{-1} v_{\mathrm{rel}}^{-1}\left(k_{\mathrm{B}} T_{0} / m\right)^{\frac{1}{2}}\left[\Im\left(\begin{array}{c}
0200 \\
0200
\end{array}\right) \subseteq\left(\begin{array}{l}
1200 \\
1200
\end{array}\right)\right]^{-\frac{1}{2}} \\
& =n_{0}{ }^{-1} \frac{1}{4} \sqrt{\pi}\left[\Im\left(\begin{array}{c}
0200 \\
0200
\end{array}\right) \Im\left(\begin{array}{l}
1200 \\
1200
\end{array}\right)\right]^{-\frac{1}{2}},
\end{aligned}
$$

somehow characterizes the thickness of the layer in which the transition from the boundary value to the bulk value of tensor polarization takes place.

The solution of Eq. (33),

$$
\left.\mathbf{A}_{n}{ }^{(02)}=\frac{2 z}{1+i n \varphi_{02}}{ }_{\left(\boldsymbol{e}_{z} \boldsymbol{\nabla} p^{(0)}\right.}\right)_{n}+\mathbf{B}_{n},
$$

is the sum of the bulk value of tensor polarization, first term in Eq. (35), and of a "boundary layer" term $\mathbf{B}_{n}$ which satisfies the homogeneous differential equation

$$
\left(1-L_{n}^{2} \frac{\mathrm{d}^{2}}{\mathrm{~d} z^{2}}\right) \mathbf{B}_{n}=\mathbf{O} .
$$

Consequently, $\mathbf{B}_{n}$ will be the superposition of a $\sinh \left(z / L_{n}\right)$ and a $\cosh \left(z / L_{n}\right)$ term. For the inhomogeneity in the boundary condition (29) one has, for $z= \pm d, \boldsymbol{k}_{0}{ }^{\tan }=-d \boldsymbol{\nabla} p^{(0)}$ and $\boldsymbol{n}= \pm \boldsymbol{e}_{z}$, so that $\mathbf{A}_{n}{ }^{(02)}$ has to be an odd function of $z$. Hence, the solution for $\mathbf{B}_{n}$ can be written in the form

$$
\left.\mathbf{B}_{n}=\beta_{n} \frac{\sinh \left(z / L_{n}\right)}{\sinh \left(d / L_{n}\right)} 2 d \overline{\left(\boldsymbol{e}_{z} \boldsymbol{\nabla} p^{(0)}\right.}\right)_{n},
$$

and with the ansatz for the Kagan polarization,

$$
\begin{aligned}
\boldsymbol{e}_{z} \cdot \underline{\mathbf{A}}^{(12)} & =-L_{0} \sum_{n=-2}^{+2} \frac{1}{1+i n \varphi_{12}} \\
\cdot & {\left[\frac{\mathrm{d}}{\mathrm{d} z} \mathbf{A}^{(02)}+(1+\varepsilon) f_{1} \overline{\boldsymbol{e}_{z} \boldsymbol{\nabla} p^{(0)}}\right]_{n}, }
\end{aligned}
$$

the constant $\beta_{n}$ is found from the boundary condition (29) as

$$
\beta_{n}=-\left(\frac{1}{1+i n \varphi_{02}}-\tilde{C}_{\mathrm{ma}}\right) /\left(1+\tilde{C}_{\mathrm{a}} \sqrt{\frac{1+i n \varphi_{02}}{1+i n \varphi_{12}}}\right) .
$$

This actually is the simplest approximation for $\beta_{n}$, neglecting terms of the order $L_{0} / d$ and replacing $\operatorname{coth}\left(d / L_{n}\right)$ by 1 .

According to Eq. (36), the surface contribution $\mathbf{B}_{n}$ to the total tensor polarization profile is different from zero only in a boundary layer of thickness $L_{0}$. Further away from the wall, only the bulk contribu. tion, first term in Eq. (35), remains. For the value of the tensor polarization at the plates,

$$
\begin{aligned}
& \mathbf{A}_{n}{ }^{(02)}(d)=-\mathbf{A}_{n}{ }^{(02)}(-d) \\
& =\left[\frac{1}{1+i n \varphi_{02}}+\beta_{n}\right] 2 d\left(\widehat{\boldsymbol{e}_{z} \boldsymbol{\nabla} p^{(0)}}\right)_{n}
\end{aligned}
$$

both contributions may be of the same order of magnitude. In Levi, McCourt and Beenakker's approximation ${ }^{6}$, only the bulk term [first term in Eq. (39) ] was taken into account. Neglecting the surface contribution is, from the present point of view, equivalent to assuming large $\tilde{C}_{\mathrm{a}}$-values, $\tilde{C}_{\mathrm{a}} \gg 1$ and $\tilde{C}_{\mathrm{a}} \gg\left|\tilde{C}_{\mathrm{ma}}\right|$; realistic values of $\tilde{C}_{\mathrm{a}}$, however, are of the order of unity ${ }^{12,26}$. In order to get some feeling for the relation between the size of the wall effects and the magnitude of the surface parameter $\tilde{C}_{\mathrm{ma}}$, two special cases of Eq. (39) are discussed now. For zero field, the "surface contribution" $\beta_{0}=$ $\left(\tilde{C}_{\mathrm{ma}}-1\right) /\left(\tilde{C}_{\mathrm{a}}+1\right)$ has to be compared with the "bulk value" of 1 . With $\tilde{C}_{\mathrm{ma}}<1$, one gets $\beta_{0}<0$, i. e. different types of tensor polarization are produced in the boundary layer and in the bulk gas. For high fields, the bulk contribution vanishes, whereas the surface contribution is proportional to

$$
\beta_{n}=\tilde{C}_{\mathrm{ma}} /\left(1+\tilde{C}_{\mathrm{a}} \sqrt{\left.\widetilde{\Im}\left(\begin{array}{c}
1200 \\
1200
\end{array}\right) / \widetilde{S}\left(\begin{array}{l}
0200 \\
0200
\end{array}\right)\right)} .\right.
$$

Hence, the sign of $\check{C}_{\mathrm{ma}}$ determines the type of tensor polarization which survives near the wall. More details about the tensor polarization profile (35), (36) can be found in Reference ${ }^{26}$.

\section{The Magnetic Field Dependent Pressure Gradient}

The complete expression for the pressure gradient is obtained from the solution for plane Poiseuille flow in a magnetic field ${ }^{26}$. In the limit $L_{0} / d=0$ however, the expression for $\partial p / \partial y$ can easily be derived, using the following arguments. Basically, one assumes that $\nabla p$ is a function of $z$ only, then $\partial p / \partial x$ and $\partial p / \partial y$ must be constant. By insertion of the ansatz for the friction pressure tensor

$$
\overline{\mathbf{p}}=-2 \eta^{[0]}\left(\overline{\boldsymbol{\nabla} \boldsymbol{v}}+\frac{2}{5}(1+\varepsilon) p_{0}{ }^{-1} \overline{\boldsymbol{\nabla} \boldsymbol{q}_{\mathrm{tr}}}\right)-\Psi^{(\eta)} \mathbf{A}^{(02)}
$$

into the steady state equation for momentum conservation,

$$
\boldsymbol{\nabla} p=-\boldsymbol{\nabla} \cdot \mathbf{p},
$$

one readily finds, using $\boldsymbol{\nabla} \cdot \boldsymbol{v}=0, \boldsymbol{\nabla} \cdot \boldsymbol{q}_{\mathrm{tr}}=0$,

$$
\begin{aligned}
\boldsymbol{\nabla} p= & \eta^{[0]} \boldsymbol{\nabla} \cdot \boldsymbol{\nabla}\left(\boldsymbol{v}+\frac{2}{5}(1+\varepsilon) p_{0}{ }^{-1} \boldsymbol{q}_{\mathrm{tr}}\right) \\
& +\Psi^{(\eta)} \boldsymbol{\nabla} \cdot \mathbf{A}^{(02)} .
\end{aligned}
$$


Here, $\eta^{[0]}$ denotes the "isotropic" value of shear viscosity, and the quantity $\Psi^{(\eta)}$,

$$
\Psi^{(\eta)}=\frac{\mathbb{S}^{2}\left(\begin{array}{l}
2000 \\
0200
\end{array}\right)}{\subseteq\left(\begin{array}{c}
2000 \\
2000
\end{array}\right) \subseteq\left(\begin{array}{l}
0200 \\
0200
\end{array}\right)},
$$

characterizes the relative magnitude of the viscosity field effect. The last term on the right hand side of Eq. (40) gives a constant and an exponentially varying contribution which vanishes in the bulk gas. Since the second term reduces to third order derivatives of $\mathbf{A}^{(02)}$, only the exponentials (from $\mathbf{B}_{n}$ ) remain. Finally let us look at the $y$-component of the flow velocity; $v_{y}$ will be the sum of a parabolic profile and of terms decaying exponentially with increasing distance from the walls. By the flow in $y$-direction, no gas shall leave the channel, so that $\bar{v}_{y}=0$ must be required. Consequently, in the bulk gas, $v_{y}$ is of order $L_{0} / d$. Therefore, $\boldsymbol{\nabla} \cdot \boldsymbol{\nabla} v_{y}$ contains constant terms of the order $L_{0} / d$ and exponential terms. As a conclusion one finds that the constant transverse pressure gradient $\partial p / \partial y$, for $L_{0} / d=0$, is determined by the constant terms in $\boldsymbol{\nabla} \cdot \mathbf{A}^{(\mathbf{0 2})}$ only, hence from Eqs. (35), (40)

$$
\frac{\partial p}{\partial y}=\Psi^{(\eta)} \sum_{n=-2}^{+2} \frac{2}{1+i n \varphi_{02}}\left(\boldsymbol{e}_{y} \boldsymbol{e}_{z}\right):\left[\overline{\boldsymbol{e}_{z} \boldsymbol{\nabla} p^{(0)}}\right]_{n} .
$$

\section{The Transverse Temperature Gradient}

By insertion of the results (39) and (42) for $\mathbf{A}^{(02)}(d)$ and $\partial p / \partial y$ into Eq. (19), the $y$-component of the average viscomagnetic heat flux in the bulk is obtained as

$$
\begin{aligned}
\boldsymbol{e}_{y} \cdot \overline{\boldsymbol{q}}_{\mathrm{VM}}= & \lambda T_{0} p_{0}{ }^{-1}\left\{-\alpha \Psi_{1} \boldsymbol{e}_{y} \cdot \boldsymbol{\Lambda}\left(\varphi_{12}\right) \cdot \boldsymbol{\nabla} p^{(0)}\right. \\
& +\sum_{n=-2}^{+2} 2\left(\boldsymbol{e}_{y} \boldsymbol{e}_{z}\right):\left[\widetilde{\boldsymbol{e}_{z} \boldsymbol{\nabla} p^{(0)}}\right]_{n} \\
\cdot & {\left.\left[\frac{\alpha \Psi^{(\eta)}}{1+i n \varphi_{02}}+\frac{\Psi_{2}}{1+i n \varphi_{12}}\left(\frac{1}{1+i n \cdot \varphi_{02}}+\beta_{n}\right)\right]\right\} . }
\end{aligned}
$$

The term with the $\beta_{n}$ is the surface contribution, whereas the three remaining terms constitute the bulk contribution already given by Levi, McCourt and Beenakker ${ }^{6}$.

In addition to the bulk heat flux $\boldsymbol{q}_{\mathrm{VM}}$ there is also the surface heat flux $\mathbf{Q}$ which is given by the boundary condition (32). For the flow between infinite parallel plates, the first term in Eq. (32) is simply found from Eq. (42) and from

$$
\boldsymbol{k}^{\tan }(d)=\boldsymbol{k}^{\tan }(-d)=-d(\boldsymbol{\nabla} p)^{\tan } .
$$

The second term in Eq. (32) is calculated with the help of the ansatz (37) for the Kagan polarization. Here, in lowest order in $L_{0} / d$, only the exponential $\mathbf{B}_{n}$-term in $\mathbf{A}^{(02)}$ has to be retained in Eq. (37) ; in the same approximation, $\operatorname{coth}\left(d / L_{n}\right)$ is replaced by 1 . The result for the $y$-component of the surface heat flux is

$$
\begin{aligned}
Q_{y}(d) & =Q_{y}(-d) \\
& =d \lambda T_{0} p_{0}{ }^{-1} \sum_{n=-2}^{+2} 2\left(\boldsymbol{e}_{y} \boldsymbol{e}_{z}\right):\left(\widetilde{\boldsymbol{e}_{z} \boldsymbol{\nabla} p^{(0)}}\right)_{n} \\
& \cdot\left[\frac{\sigma \Psi^{(\eta)}}{1+i n \varphi_{02}}+\tilde{C}_{\mathrm{ha}} \Psi_{2} \beta_{n} \sqrt{\frac{1+i n \varphi_{02}}{1+i n \varphi_{12}}}\right] .
\end{aligned}
$$

Now, with Eqs. (43), (44) the general expression for the transverse temperature gradient $\partial T / \partial y$ can be derived from Equation (5). Of practical interest ${ }^{9}$ in particular is the case where the magnetic field varies in the plane perpendicular to the field-free flow direction $\boldsymbol{e}_{x}$,

$$
\boldsymbol{h}=\cos \chi \boldsymbol{e}_{y}+\sin \chi \boldsymbol{e}_{z} .
$$

Using Eq. (38) for $\beta_{n}$, and evaluating the dependence on the field direction [with Eq. (12) and the Appendix of Ref. ${ }^{26}$ ] with $\boldsymbol{\nabla} p^{(0)}=\boldsymbol{e}_{x}(\partial p / \partial x)_{0}$ leads to the explicit result:

$\frac{\partial T}{\partial y}=-\frac{T_{0}}{p_{0}}\left(\frac{\partial p}{\partial x}\right)_{0} \sin \chi\left[\delta+\Delta_{1}+\cos ^{2} \chi\left(\Delta_{2}-2 \Delta_{1}\right)\right]$,

where

$$
\begin{aligned}
& \delta=\alpha \Psi_{1} \frac{1}{2}\left(g\left(\varphi_{12}\right)+2 g\left(2 \varphi_{12}\right)\right), \\
& \Delta_{n}=\left((\sigma+\alpha) \Psi^{(\eta)}+\frac{r}{r-1} \Psi_{2}\right) g\left(n \varphi_{02}\right) \\
& -\frac{1}{r-1} \Psi_{2} g\left(n \varphi_{12}\right) \\
& +\Psi_{2} \operatorname{Im}\left[\frac{\frac{1}{1+i n \cdot \varphi_{02}}-\tilde{C}_{\mathrm{ma}}}{1+\tilde{C}_{\mathrm{a}} \sqrt{\frac{1+i n \varphi_{02}}{1+i n \cdot \varphi_{12}}}}\right. \\
& \left.\cdot\left(\frac{1}{1+i n \varphi_{12}}+\tilde{C}_{\mathrm{ha}} \sqrt{\frac{1+i n \varphi_{02}}{1+i n \varphi_{12}}}\right)\right], n=1,2 \text {, }
\end{aligned}
$$

and $r$ is an abbreviation for the ratio

$$
r=\Im\left(\begin{array}{l}
1200 \\
1200
\end{array}\right) / \Im\left(\begin{array}{l}
0200 \\
0200
\end{array}\right) .
$$

According to Eq. (46), the transverse temperature gradient is inversely proportional to the average gas pressure $p_{0}$, and is proportional to the field- 
free pressure gradient $(\partial p / \partial x)_{0}$. If the magnetic field is perpendicular to the plates (and to the field. free flow direction $\left.\boldsymbol{e}_{x}\right), \chi=90^{\circ}$, the temperature gradient as a function of $H / p_{0}$ reaches its largest absolute value if $\left|\varphi_{02}\right|,\left|\varphi_{12}\right|$ are about unity. With decreasing angle $\chi$, this maximum decreases about like $\sin \chi$ and shifts to lower $H / p_{0}$-values, since for $\chi<90^{\circ}$ the double frequencies $2 \varphi_{02}, 2 \varphi_{12}$ (through $\Delta_{2}$ ) come into play. The transverse pressure gradient [according to Eq. (42)],

$$
\begin{aligned}
\frac{\partial p}{\partial y}= & -\left(\frac{\partial p}{\partial x}\right)_{0} \Psi^{(\eta)} \sin \chi \\
& \cdot\left[g\left(\varphi_{02}\right)+\cos ^{2} \chi\left(g\left(2 \varphi_{02}\right)-2 g\left(\varphi_{02}\right)\right)\right],
\end{aligned}
$$

shows the same type of angular dependence. Notice, that the first terms in Eq. (49) and in $\Delta_{1}$ have the magnetic field dependence of the viscosity coefficient $\eta_{5}$,

$$
\eta_{5}=-\eta \Psi^{(\eta)} g\left(\varphi_{02}\right),
$$

and that the quantity $\delta$ is proptortional to the transverse heat conductivity coefficient

$$
\lambda^{\text {transv }}=-\lambda \Psi^{(\lambda)} \frac{1}{2}\left(g\left(\varphi_{12}\right)+2 g\left(2 \varphi_{12}\right)\right) .
$$

For $\chi=90^{\circ}$, the maximum of $|\partial T / \partial y|$ as a function of $H / p_{0}$ will therefore occur somewhere between that of $\left|\eta_{5}\right|$ and of $\left|\lambda^{\text {transv }}\right|$.

Now let us look more closely into some details of Eqs. (47) and (48). The $\delta$-term and the second, third and fourth terms in $\Delta_{n}$ are the bulk contributions already given by Levi, McCourt and Beenak$\operatorname{ker}^{6}$. Their result is obtained from our general expression (47), (48) with $\varepsilon=0$ [i. e. $\alpha=\frac{2}{5} \lambda_{\text {tr }} / \lambda$, see Eq. (9) ], $\sigma=0$ and $\tilde{C}_{\mathrm{ma}}=0=\tilde{C}_{\mathrm{ha}}, \tilde{C}_{\mathrm{a}} \gg 1$. The remaining terms in $\Delta_{n}$ are the surface contributions, two of them originating from the surface heat flux (proportional to $\sigma$ and $\tilde{C}_{\mathrm{ha}}$ ), and the others (involving $\tilde{C}_{\mathrm{a}}$ and $\tilde{C}_{\mathrm{ma}}$ ) from the wall effects for the tensor polarization. The relative importance of the surface contributions is determined by the values for the bulk constants $\Psi_{1}, \Psi_{2}, \Psi^{(\eta)}$, and by those for the surface parameters $\tilde{C}_{\mathrm{a}}, \tilde{C}_{\mathrm{ma}}, \tilde{C}_{\mathrm{ha}}$. The constants $\Psi_{1}, \Psi_{2}$ and $\Psi^{(\eta)}$ can be calculated from Eqs. (16) - (18) and (41) with the values for effective cross sections derived from sound absorption measurements (rotational relaxation, $\eta_{\mathrm{v}}$ ) and from data on the field effects of viscosity and thermal conductivity, see e. g. the values compiled in Reference ${ }^{27}$. Thus, the bulk contributions to $\partial T / \partial y$ are known, see Table I. The two surface parameters $\tilde{C}_{\mathrm{a}}$ and $\tilde{C}_{\mathrm{ma}}$ determine the magnitude of the Knudsen corrections for the viscosity field effect ${ }^{26}$ and for flow birefringence ${ }^{28}$. Furthermore, they enter into the theoretical expression for the thermomagnetic pressure difference ${ }^{13}$. From this information, sets of $\tilde{C}_{\mathrm{a}}$ and $\tilde{C}_{\mathrm{ma}}$ can be found for these gases ${ }^{12}$.

In the next section, it will be shown how the value of the yet unknown parameter $\tilde{C}_{\mathrm{ha}}$ influences the magnitude of the transverse temperature gradient $\partial T / \partial y$, the shape of the curve as a function of $H / p_{0}$, and the dependence on the angle $\chi$.

\section{Quantitative Results}

For the following discussions and graphs it is convenient to use the reduced temperature gradient $\tau$ instead of $\partial T / \partial y$ itself:

$$
\begin{aligned}
\tau & \equiv-\frac{p_{0}}{T_{0}} \frac{\partial T}{\partial y} /\left(\frac{\partial p}{\partial x}\right)_{0} \\
& =\sin \chi\left[\delta+\Delta_{1}+\cos ^{2} \chi\left(\Delta_{2}-2 \Delta_{1}\right)\right] .
\end{aligned}
$$

The dimensionless quantity $\tau$ is independent of the average pressure $p_{0}$ and of the field-free pressure gradient $(\partial p / \partial x)_{0}$.

First let us consider the case of $\chi=90^{\circ}$ where the magnetic field is perpendicular to the plates and to the field-free flow direction $\boldsymbol{e}_{x}$. According to Eqs. (47), (48), the bulk contribution $\tau^{\mathrm{b}}$ to the total

Table I. Values for the gas parameters $\varepsilon, \lambda_{\text {tr }} / \lambda, \alpha, \Psi_{1}, \Psi_{2}, \Psi(\eta)$ calculated from Eqs. (9) - (11), (16) - (18) with the help of Ref. ${ }^{22}$ and with the experimental data listed in Ref. ${ }^{27}$. The bulk contribution $\tau$ b to the reduced temperature gradient (for $\chi=90^{\circ}$ ) has its maximum value $\left|\tau^{\mathrm{b}}\right|_{\max }$ at $\left(H / p_{0}\right) \underset{\max }{\mathrm{b}}$, see Eqs. (50), (51).

\begin{tabular}{lccllllll}
\hline & $\varepsilon$ & $\frac{\lambda}{\operatorname{tr}}$ & $\alpha$ & $10^{3} * \Psi_{1}$ & $10^{3} * \Psi_{2}$ & $10^{3 * \Psi(\eta)}$ & $\begin{array}{l}\left(H / p_{0}\right)_{\max }^{\mathrm{b}} \\
\mathrm{kOe} / \text { torr }\end{array}$ & $10^{3 *}\left|\tau^{\mathrm{b}}\right|_{\max }$ \\
\hline $\mathrm{HD}$ & -0.14 & 0.715 & 0.246 & 0.50 & 1.61 & 1.97 & 0.47 & 1.20 \\
$\mathrm{CH}_{4}$ & -0.07 & 0.609 & 0.226 & 0.87 & 0.34 & 0.80 & 5.26 & 0.44 \\
$\mathrm{CO}$ & -0.09 & 0.720 & 0.262 & 4.49 & 1.50 & 3.52 & 4.39 & 2.23 \\
$\mathrm{~N}_{2}$ & -0.11 & 0.715 & 0.254 & 3.69 & 1.53 & 2.72 & 3.49 & 1.94 \\
\hline
\end{tabular}


reduced temperature gradient $\tau$ is given by

$$
\begin{aligned}
\tau^{\mathrm{b}}= & \left(\alpha \Psi^{(\eta)}+\frac{r}{r-1} \Psi_{2}\right) g\left(\varphi_{02}\right) \\
& -\frac{1}{r-1} \Psi_{2} g\left(\varphi_{12}\right)+\delta .
\end{aligned}
$$

The total effect also contains the surface contribution $\tau^{\mathrm{s}}, \tau=\tau^{\mathrm{b}}+\tau^{\mathrm{s}}$. For the gases $\mathrm{HD}, \mathrm{CH}_{4}, \mathrm{CO}$ and $\mathrm{N}_{2}$, the bulk contribution has the same sign as the rotational $g$-factor, viz. $\tau^{\mathrm{b}}>0$ for $\mathrm{HD}, \mathrm{CH}_{4}$, and $\tau^{\mathrm{b}}<0$ for $\mathrm{CO}, \mathrm{N}_{2}$. The maximum value of $\left|\tau^{\mathrm{b}}\right|$ is of the order $10^{-3}$, see Table I. For $\mathrm{N}_{2}$ and HD, the bulk contribution is plotted as a function of $H / p_{0}$ in Figs. 1, 2, together with the surface contributions and the total effect for a set of $\tilde{C}_{\mathrm{a}}, \tilde{C}_{\mathrm{ma}}$ (see Ref. ${ }^{12}$ ) and three values of $\tilde{C}_{\mathrm{ha}}$. In the case of $\tilde{C}_{h \mathrm{a}}=0$, the surface contributions subtract from the bulk contributions, viz. for $\mathrm{N}_{2}$ over the whole $H / p_{0^{-}}$ range, and for $\mathrm{HD}$ at lower $H / p_{0}$-values (i.e. $H / \mathrm{p}_{0} \leqq 2 \mathrm{kOe} /$ torr $)$. With large negative values of $\tilde{C}_{\text {ha }}$, however, the surface contributions add to the bulk contributions, e.g. for $\tilde{C}_{\mathrm{ha}}=-3.0$ in the case of $\mathrm{N}_{2}$ (see Figure 1). This behaviour can be qualitatively understood from the discussion of the two limits $\varphi_{p q} \rightarrow 0$ and $\varphi_{p q} \rightarrow \infty$ of Eqs. (47) and (48).

For $\left|\varphi_{p q}\right| \ll 1$ one finds

$$
\begin{aligned}
\tau^{\mathrm{b}} \approx & \varphi_{12}\left\{\frac{5}{2} \alpha \Psi_{1}+(r+1) \Psi_{2}+\alpha r \Psi^{(\eta)}\right\}, \\
\tau^{\mathrm{s}} \approx & \varphi_{12}\left\{\sigma r \Psi^{(\eta)}-\frac{\Psi_{2}}{\left(\tilde{C}_{\mathrm{a}}+1\right)^{2}}\right. \\
& \cdot\left[r+1+\frac{3 r+1}{2} \tilde{C}_{\mathrm{a}}-\tilde{C}_{\mathrm{ma}}\left(1+\frac{r+1}{2} \tilde{C}_{\mathrm{a}}\right)\right. \\
& \left.\left.+\tilde{C}_{\mathrm{ha}}\left(\frac{r+1}{2}+r \tilde{C}_{\mathrm{a}}+\frac{r-1}{2} \tilde{C}_{\mathrm{ma}}\right)\right]\right\} .
\end{aligned}
$$

In the case of $\tilde{C}_{\mathrm{ha}}=0, \tau^{\mathrm{s}}$ would have the same sign as $\tau^{\mathrm{b}}$ only for large positive values of $\tilde{C}_{\mathrm{ma}}\left(\tilde{C}_{\mathrm{a}}>0\right.$, and usually $\sigma<0)$; e.g. for $\mathrm{N}_{2}(r=2.0)$ if $\tilde{C}_{\text {ma }}$ $>2.6$, and for HD $(r=6.1)$ if $\tilde{C}_{\mathrm{ma}}>0$. With the $\tilde{C}_{\mathrm{ma}}$-values used in Figs. 1, 2, $\tau^{\mathrm{s}} \varphi_{12}^{-1}$ is negative, whereas $\tau^{\mathrm{b}} \varphi_{12}^{-1}$ is positive. Negative values of $\tilde{C}_{\mathrm{ha}}$ increase $\tau^{\mathrm{s}} \varphi_{12}^{-1}$ and make it finally positive, viz. for $\tilde{C}_{\text {ha }}<-2.43$ in the case of $\mathrm{N}_{2}$, and for $\tilde{C}_{\mathrm{ha}}<-1.51$ in the case of HD.

In the limit $\left|\varphi_{p q}\right| \gg 1$ on the other hand, one finds

$$
\begin{aligned}
& \tau^{\mathrm{b}} \approx \varphi_{12}^{-1} \alpha\left(\Psi_{1}+r^{-1} \Psi^{(\eta)}\right), \\
& \tau^{\mathrm{s}} \approx \varphi_{12}^{-1}\left[r^{-1} \sigma \Psi^{(\eta)}+\Psi_{2}\left(\tilde{C}_{\mathrm{ma}}-\sqrt{r^{-1}} \tilde{C_{\mathrm{ha}}}\right)\right. \\
& \left.\quad /\left(1+\sqrt{r} \tilde{C_{\mathrm{a}}}\right)\right] .
\end{aligned}
$$

Here $\tau^{\mathrm{b}} \varphi_{12}$ is positive, whereas for $\tilde{C}_{\mathrm{ha}}=0$ and $\tilde{C}_{\mathrm{ma}}<0$ (e. g. $\left.\mathrm{N}_{2}\right) \tau^{\mathrm{s}} \varphi_{12}$ is negative; for HD with $\tilde{C}_{\mathrm{ma}}=0.4$ and $\tilde{C}_{\mathrm{ha}}=0, \tau^{\mathrm{s}} \varphi_{12}$ is positive (see Figs. 1 and 2). Again, negative $\hat{C}_{\mathrm{ha}}$-values increase $\tau^{\mathrm{s}} \varphi_{12}$, e. g. for $\mathrm{N}_{2}, \tau^{\mathrm{s}} \varphi_{12}$ gets positive if $\tilde{C}_{\mathrm{ha}}<-0.62$.

With these considerations one can qualitatively understand Figs. 1 and 2. Furthermore, one can see that the total effect $(\tau)$ increases with increasing $\tilde{C}_{\mathrm{ma}}$ and decreasing $\tilde{C}_{\mathrm{ha}}$. The absolute value of the surface contribution $\tau^{\S}$ decreases with increasing $\tilde{C}_{\mathrm{a}}$. The dependence of $\tau$ on $\tilde{C}_{\mathrm{a}}$ and $\tilde{C}_{\mathrm{ma}}$ is in accordance with that of the tensor polarization discussed in Section 5.

From the preceding discussion it is obvious that for $\tilde{C}_{\mathrm{ha}} \neq 0$ the shape of the total curve $\tau=\tau\left(H / p_{0}\right)$ will change considerably compared with the case of $\tilde{C}_{\mathrm{ha}}=0$, or with the bulk curve $\tau^{\mathrm{b}}$. In order to clearly demonstrate the effect of the surface contributions on the shape of the curve $\tau=\tau\left(H / p_{0}\right)$, the dimensionless ratio $Q$,

$$
\varrho=\frac{p_{0}}{T_{0}} \frac{\partial T}{\partial y} /\left(\frac{\partial p}{\partial y}\right)
$$

of the transverse temperature gradient and the transverse pressure gradient is plotted versus $H / p_{0}$ in Figs. 3 and 4 for $\mathrm{N}_{2}$ and HD, respectively. For both gases, $Q$ is positive and of order unity. The surface contributions influence the value of $\varrho$ in a different way at low and high fields; e.g. for the bulk contributions alone, $\varrho$ decreases at higher $H / p_{0}$, whereas the inclusion of the surface contributions may result in an increase of $\varrho$.

With the parameters used here, the total transverse temperature gradient for $\chi=90^{\circ}$ has the same

Fig. 1. Bulk $(\cdots)$ and surface $(--)$ contributions to the total (-) reduced temperature gradient $\tau$ for $\mathrm{N}_{2}$, calculated for $\chi=90^{\circ}$ from Eqs. (47), (48), (50), (51) with the help of Table I, $\tilde{C_{\mathrm{a}}}=1.0, \tilde{C_{\mathrm{ma}}}=-0.3$ and three $\tilde{C_{\mathrm{ha}}}$ values. The effective thermal slip number $\sigma+\alpha=0.189$ was taken from Ref. ${ }^{16}$.

Fig. 2. Same as Fig. 1 for the gas $\mathrm{HD}$ with $\tilde{C}_{\mathrm{a}}=0.4, \tilde{C}_{\mathrm{ma}}=$ $0.4, \sigma+a=0.2$.

Fig. 3. The ratio $\varrho=\frac{p_{0}}{T_{0}} \frac{\partial T}{\partial y} /\left(\begin{array}{c}\partial p \\ \partial y\end{array}\right)$ for $\mathrm{N}_{2}$, calculated for $\chi=90^{\circ}$ from Eqs. (46)-(49) with the parameters from Fig. $1 ; \cdots$ is the bulk contribution, - is the total effect for three $\tilde{C}_{\mathrm{ha}}$ values.

Fig. 4. Same as Fig. 3 for HD with parameters from Figure 2. 

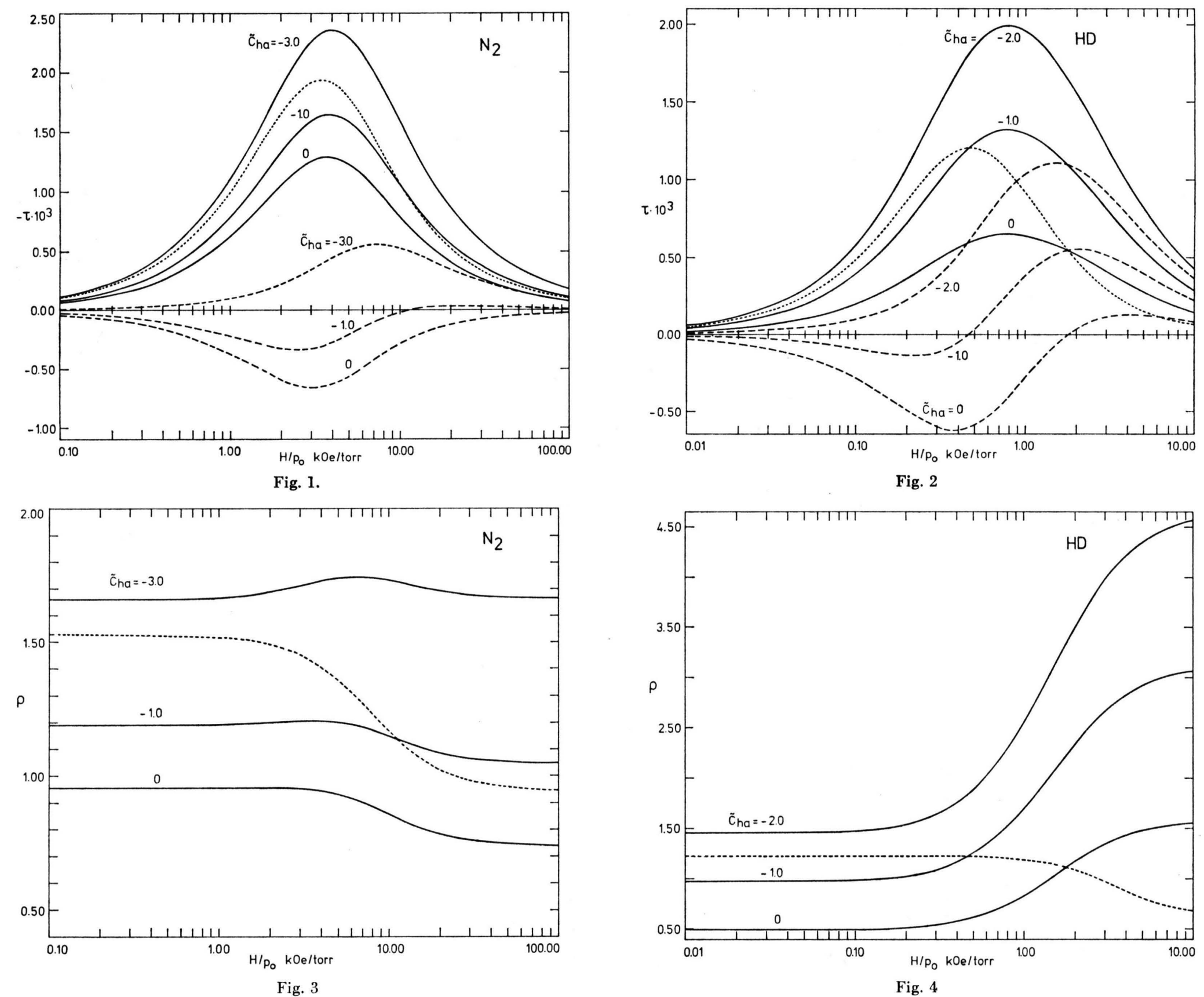


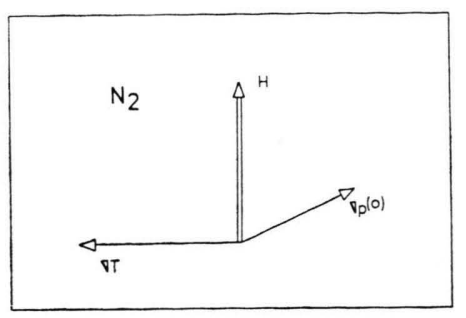

Fig. 5. Direction of the transverse temperature gradient $\boldsymbol{e}_{y} \frac{\partial T}{\partial y}$ for $\mathrm{N}_{2}\left(g_{\mathrm{r}}<0\right)$, with the magnetic field $\boldsymbol{H}=\boldsymbol{e}_{z} H$ $\left(\chi=90^{\circ}\right)$ and the field free pressure gradient $\nabla p^{(0)}=$

$$
\boldsymbol{e}_{x}\left(\frac{\partial p}{\partial x}\right)_{0}
$$

direction as the bulk contribution, viz. that of

$$
\operatorname{sign}\left(g_{\mathrm{r}}\right) \boldsymbol{\nabla} p^{(\boldsymbol{0})} \times \boldsymbol{h} .
$$

The relation between the three vectors $\boldsymbol{e}_{y} \partial T / \partial y$, $\boldsymbol{\nabla} p^{(0)}$ and $\boldsymbol{h}$ is shown in Fig. 5 for $\mathrm{N}_{2}$.

Finally, the angular dependence of the effect shall be discussed, i. e. the change in $\partial T / \partial y$ through the variation of the field direction $\boldsymbol{h}$ in the plane perpendicular to the field-free flow direction $\boldsymbol{e}_{x}$. If the angle $\chi$ gets smaller than $90^{\circ}$, the absolute height of the effect decreases, and the maximum occurs at lower $H / p_{0}$-values. This can be easily understood by inspection of Eq. (50): $|\tau|$ decreases with about $\sin \psi$, and the double frequencies $2 \varphi_{p q}$ become important at $\chi<90^{\circ}$. In Fig. 6 , the change of the whole $\tau\left(H / p_{0}\right)$ curve with the angle $\chi$ is shown for $\mathrm{N}_{2}$ and four $\chi$-values. For the same gas, Figs. 7 and 8 display the dependence on $\chi$ of the value $|\tau|_{\max }$ and the position $\left(H / p_{0}\right)_{\max }$ of the maximum of $|\tau|$, both normalized with the $\chi=90^{\circ}$ values. The $\chi=90^{\circ}$ values of $|\tau|_{\max }$ and $\left(H / p_{0}\right)_{\max }$ for $\mathrm{N}_{2}$ are listed in Table II for $\tilde{C}_{\mathrm{a}}=1.0$, two "reasonable" values ${ }^{12}$ of $\tilde{C}_{\mathrm{ma}}$ and four $\tilde{C}_{\mathrm{ha}}$ values. The maximum value of $|\tau|$ increases with increasing $\tilde{C}_{\mathrm{ma}}$ and decreasing $\tilde{C}_{\mathrm{ha}}$ and shifts to higher $H / p_{0}$ values. From $|\tau|_{\max },|\partial T / \partial y|_{\max }$ is easily obtained by observing that, with $T_{0}=300 \mathrm{~K}, p_{0}=1$ torr and $(\partial p / \partial x)_{0}=0.1$ torr $/ \mathrm{cm}$, a value of $\tau=10^{-3}$ corresponds to $\partial T / \partial y=30 \mathrm{mK} / \mathrm{cm}$.

Table II. Maximum position $\left(H / p_{0}\right)_{\max }$ and value $|\tau|_{\max }$ of the reduced temperature gradient $\tau$ for $\mathrm{N}_{2}$ at $\chi=90^{\circ}$, calculated from Eqs. (47), (48), (50) with the parameters listed in Table I, with $\tilde{C}_{\mathrm{a}}=1.0$ and two $\tilde{C}_{\mathrm{ma}}$-values ${ }^{12}$, for four different $\tilde{C}_{\mathrm{ha}}$. The effective thermal slip number $\sigma+\alpha$ $=0.189$ was taken from thermal transpiration data ${ }^{16}$.

\begin{tabular}{cllll}
\hline & \multicolumn{2}{c}{$\tilde{C}_{\mathrm{ma}}=-0.3$} & \multicolumn{2}{c}{$\tilde{C}_{\mathrm{ma}}=-0.8$} \\
\hline$\tilde{C}_{\mathrm{ha}}$ & $\begin{array}{c}\left(H / p_{0}\right)_{\max } \\
\mathrm{k} O e / \text { torr }\end{array}$ & $10^{3}|\tau|_{\max }$ & $\begin{array}{l}\left(H / p_{0}\right)_{\max } \\
\mathrm{kOe} / \text { torr }\end{array}$ & $10^{3}|\tau|_{\max }$ \\
\hline 0 & 3.77 & 1.29 & 3.56 & 1.13 \\
-1.0 & 3.89 & 1.64 & 3.71 & 1.45 \\
-3.0 & 4.05 & 2.36 & 3.88 & 2.09 \\
-5.0 & 4.13 & 3.07 & 3.99 & 2.74 \\
\hline
\end{tabular}

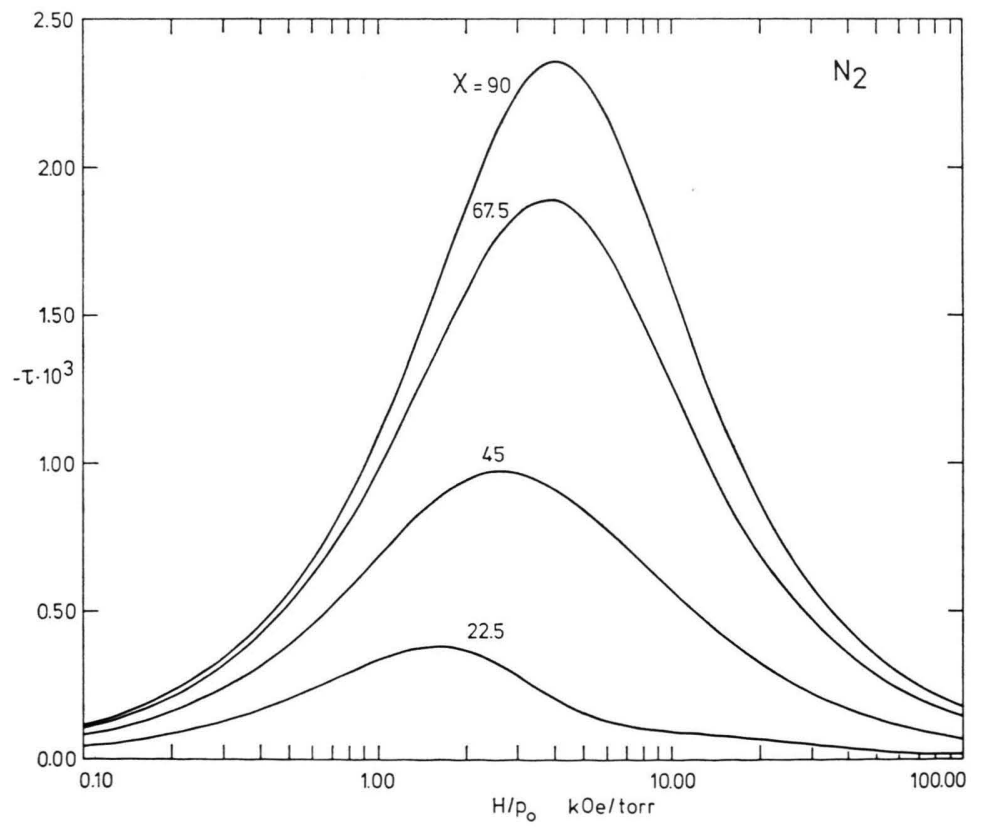

Fig. 6. Reduced transverse temperature gradient $\tau$ for $\mathrm{N}_{2}$ at four angles $\chi$ calculated from Eqs. (47), (48), (50) with $\tilde{C}_{\mathrm{a}}=1.0$, $\tilde{C}_{\mathrm{ma}}=-0.3, \tilde{C}_{\mathrm{ha}}=-3.0, \sigma+\alpha=0.189$ and the parameters from Table I. 


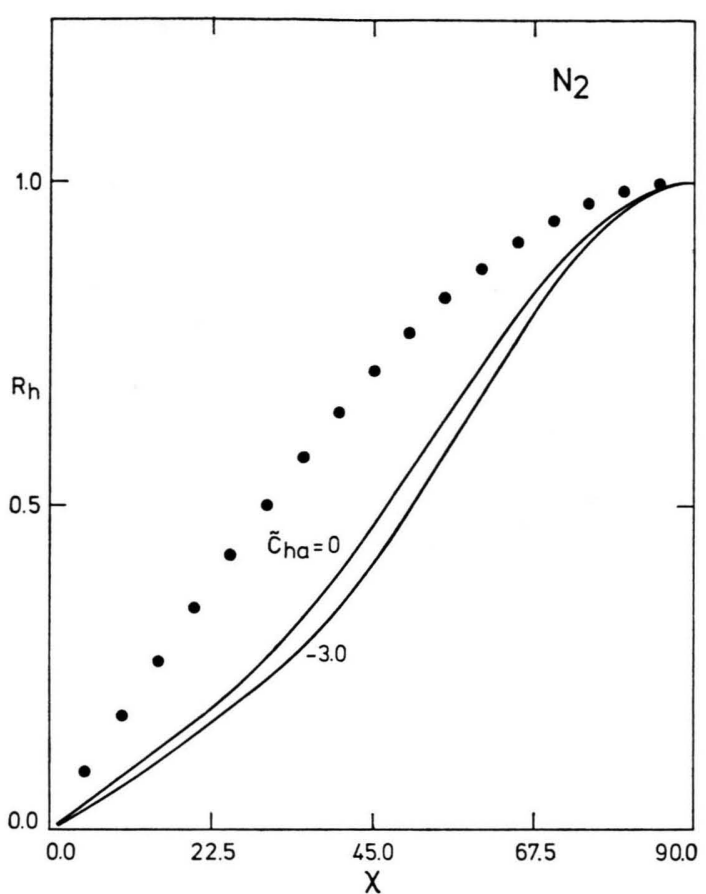

Fig. 7. Height of the maximum of $\left|\frac{\partial T}{\partial y}\right|$, normalized to the $\chi=90^{\circ}$ value, as a function of the angle $\chi$ for $\mathrm{N}_{2}, R_{\mathrm{h}}=$ $\left|\frac{\partial T}{\partial y}\right|_{\max }^{x} /\left|\frac{\partial T}{\partial y}\right|_{\max }^{90}$; the function $\sin \chi$ is indicated by $\cdots$. The parameters are $\tilde{C}_{\mathrm{a}}=1.0, \tilde{C}_{\mathrm{ma}}=-0.3, \sigma+\alpha=0.189$, $\tilde{C}_{\mathrm{ha}}=0$ and $\tilde{C}_{\mathrm{ha}}=-3.0$.

The existence of the transverse temperature gradient has been detected by Eggermont et al. ${ }^{9}$. A detailed experimental investigation of the effect is still going on. The comparison of these results with

1 J. Cl. Maxwell, Phil. Trans. 170, 231 [1879]; reprinted in Maxwell's Scientific Papers 2, 681 [Cambridge 1890].

2 S. Chapman and T. G. Cowling, The Mathematical Theory of Non-uniform Gases, Cambridge University Press 1970.

3 J. E. Jones, Phil. Trans. Roy. Soc. London A 223, 1 [1922].

4 D. Burnett, Proc. London Math. Soc. 40, 382 [1936].

5 A. C. Levi and J. J. M. Beenakker, Phys. Letters 25 A, 350 [1967].

B A. C. Levi, F. R. McCourt, and J. J. M. Beenakker, Physica 42, 363 [1969].

7 For a review see e. g. J. J. M. Beenakker and F. R. Mc Court, Ann. Rev. Phys. Chem. 21, 47 [1970]; J. J. M. Beenakker, Transport Properties in Gases in the Presence of External Fields, in Lecture Notes in Physics, Vol. 31, Springer-Verlag, Berlin 1974, p. 414.

8 G. G. Scott, H. W. Sturner, and R. M. Williamson, Phys. Rev. 158, 117 [1967] ; G. G. Scott, H. W. Sturner, and R. M. Williamson, Phys. Letters 25 A, 573 [1967].

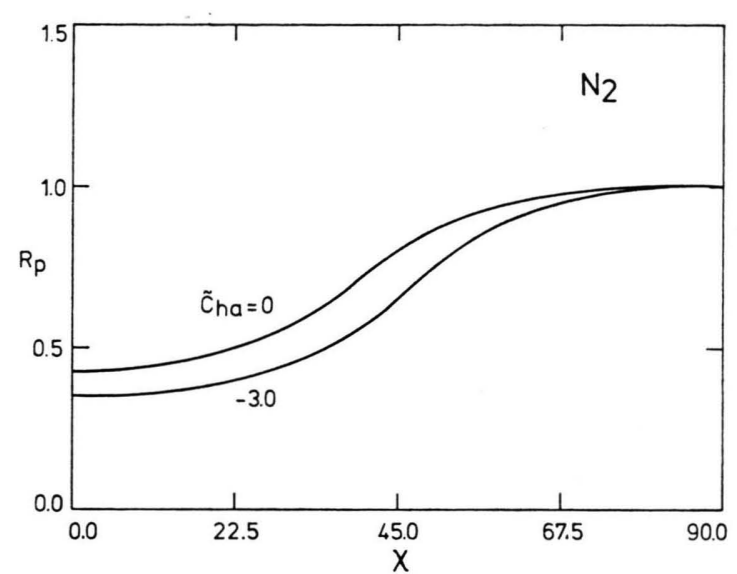

Fig. 8. Position of the maximum of $\left|\frac{\partial T}{\partial y}\right|$, normalized to the $\chi=90^{\circ}$ value, as a function of the angle $\chi$ for $\mathrm{N}_{2}, R_{\mathrm{p}}=$ $\left(H / p_{0}\right)_{\max }^{x} /\left(H / p_{0}\right)_{\max }^{90}$. The parameters are the same as in Figure 7.

the presented theory will give information on the hitherto unknown surface parameter $\tilde{C}_{\mathrm{ha}}$.

\section{Acknowledgements}

It is a pleasure to thank Prof. J. J. M. Beenakker for making possible my stay in Leiden, for his continuous interest in my work and many helpful suggestions. Thanks are due to Prof. H. F. P. Knaap, Dr. L. J. F. Hermans and Drs. G. E. J. Eggermont for numerous stimulating discussions.

This work is part of the research programme of the "Stichting voor Fundamenteel Onderzoek der Materie (F.O.M.)" and has been made possible by financial support from the "Nederlandse Organisatie voor Zuiver Wetenschappelijk Onderzoek (Z.W.O.)".

9 G. E. J. Eggermont, P. W. Hermans, L. J. F. Hermans, and J. J. M. Beenakker, Phys. Letters A, in press.

10 L. Waldmann, Z. Naturforsch. 22 a, 1678 [1967].

11 H. Hulsman, F. G. Van Kuik, H. F. P. Knaap, and J. J. M. Beenakker, Physica 57, 522 [1972] ; H. Hulsman, G. F. Bulsing, G. E. J. Eggermont, L. J. F. Hermans, and J. J. M. Beenakker, Physica 72, 287 [1974]; G. E. J. Eggermont, L. J. F. Hermans, H. F. P. Knaap, and J. J. M. Beenakker, Proc. 9th Int. Symp. Rarefied Gas Dyn., Göttingen 1974, DFVLR-Press, Porz-Wahn 1974.

12 G. E. J. Eggermont et al., Physica, to be published.

13 H. Vestner, Proc. 8th Int. Symp. Rarefied Gas Dyn., Stanford 1972, Ed. K. Karamcheti, Acad. Press, New York 1974; H. Vestner, Z. Naturforsch. 28 a, 869 [1973].

14 L. Waldmann, Z. Naturforsch. 22 a, 1269 [1967].

15 L. Waldmann and H. Vestner, Physica 80a, 523 [1975].

$16 \mathrm{H}$. Vestner and L. Waldmann, Physica, in press.

17 H. Vestner, Z. Naturforsch. 28 a, 1554 [1973]. 
18 L. Waldmann, Z. Naturforsch. 12 a, 660 [1957] ; Z. Naturforsch. 13 a, 609 [1958].

19 R. F. Snider, J. Chem. Phys. 32, 1051 [1960].

20 L. Waldmann, Z. Naturforsch. 18 a, 1033 [1963] ; S. Hess and L. Waldmann, Z. Naturforsch. 21 a, 1529 [1966] ; H. H. Raum and W. E. Köhler, Z. Naturforsch. 25 a, 1178 [1970].

$21 \mathrm{H}$. Vestner et al., Physica, to be published.

22 J. O. Hirschfelder, C. F. Curtiss, and R. B. Bird, Molecular Theory of Gases and Liquids, John Wiley and Sons, New York 1964.
23 A. C. Levi and F. R. McCourt, Physica 38, 415 [1968].

24 G. E. J. Eggermont, H. Vestner, and H. F. P. Knaap, Physica 82 a, 23 [1976].

25 S. Hess and L. Waldmann, Z. Naturforsch. 26 a, 1057 [1971].

26 H. Vestner, Z. Naturforsch. 29 a, 663 [1974].

27 J. P. J. Heemskerk, F. G. Van Kuik, H. F. P. Knaap, and J. J. M. Beenakker, Physica 71, 484 [1974].

${ }^{28}$ H. Vestner, Z. Naturforsch. 29 a, 365 [1974]. 